\title{
The study of cosmic rays with a wide-angle Cherenkov telescope
}

\section{L.V.TIMOFEEV*}

The Yu.G. Shafer Institute of Cosmophysical Research and Aeronomy

E-mail: timofeevleveikfia.ysn.ru

\section{A.A.IVANOV}

The Yu.G. Shafer Institute of Cosmophysical Research and Aeronomy

E-mail: ivanoveikfia.ysn.ru

This report presents an observation method of Cherenkov light from extensive air showers (EAS) generated by cosmic rays (CRs) above $10^{16} \mathrm{eV}$ and preliminary observations. The interest in Cherenkov light differential detectors of EAS is caused by the possibility to measure the depth of cascade maximum, Xmax, and/or the shower age via angular and temporal distributions of the Cherenkov signal. In particular, it was shown using EAS model simulations that the pulse width measured at the periphery of the shower, $r>300 \mathrm{~m}$, at sea level is pronouncedly connected with Xmax. Cherenkov detector is a wide-angle telescope working in coincidence with scintillation detectors, integral and differential Cherenkov detectors Yakutsk complex EAS. This provides the data on lateral distribution of photon intensity and total flux of Cherenkov light in EAS. The detector is located near (about $2 \mathrm{~m}$ ) with one of the integral Cherenkov detector. This detectors signal was calibrated using plastic optical radiator. So, we have a possibility to normalize an integral signal from telescope to that of the Cherenkov detector. We have completed the field testing of the prototype wide FOV telescope. A number of EAS events are detected in coincidence with the surface detectors of the Yakutsk array. A detection efficiency of the telescope is measured as well as the effective radius of the telescope detecting area. The report details the technical specifications of the telescope, as well as the results of the first experimental observations.

The 34th International Cosmic Ray Conference,

30 July- 6 August, 2015

The Hague, The Netherlands

\footnotetext{
* Speaker.
} 


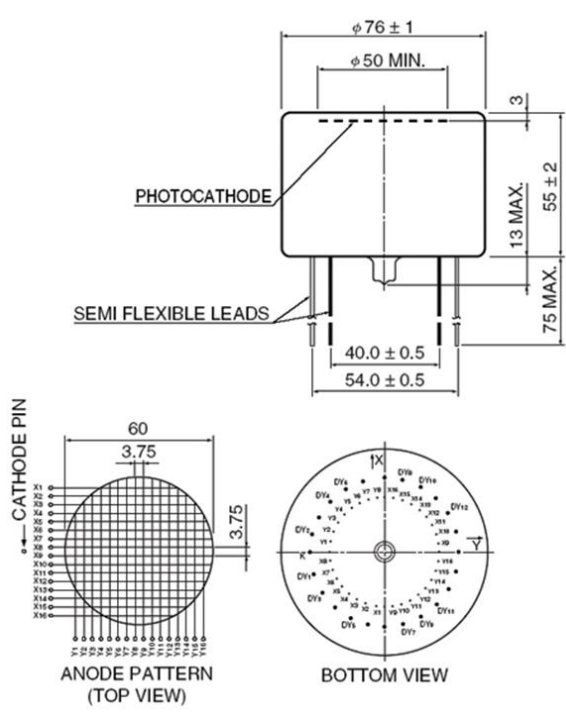

Figure 1: The photo multiplier Hamamatsu R2486 with coordinate-sensitive anode consisting of $16 \mathrm{X} 16$ crossed wires has been chosen for the Cherenkov telescope prototype.

\section{Introduction}

The current scenario of CR origin differ on the expected composition in the "knee" range and in the transition region between the galactic and extragalactic components, so the exact estimate of the average mass of the particles / nuclei in addition to the adjusted measurement range of the "knee" and the "ankle" will allow us to discard some scenarios.

Interest in the differential cherenkov light detectors for the study of EAS is due to determine the maximum depth of the cascade curve and the shower age, by means of measuring the shape of the angular and temporal distribution of the cherenkov signal. The aim of the project was to create a differential detector of cherenkov light with all-round visibility, allowing to measure the parameters of the cascade development in the atmosphere. The investigation of the galactic component of CR at the Yakutsk EAS array, supplied by the new measurements of atmospheric cherenkov radiation detector, will provide crucial experimental data to study the source of these particles, their mass composition and distribution of the arrival directions.

\section{Analogs of the Telescope and Advantages Over them}

There hasn't been measurement of EAS Cherenkov light, with respect to the angular distribution, up to now, with one exception. The project is based on the idea of creating a small copy of the giant telescopes used in gamma-ray astronomy. The latest developments of photomultiplier tubes (PMT) allows to dramatically reduce the size of the cherenkov telescope with the same angular resolution, which greatly reduces the final cost of the telescope. The energy work range $10^{16} \mathrm{eV}$ compensate reduction in the size of mirror. For the prototype Cherenkov telescope was selected photomultiplier Hamamatsu R2486 has a coordinate-sensitive anode consisting of 16X16 crossed wires (Fig. 1) . 


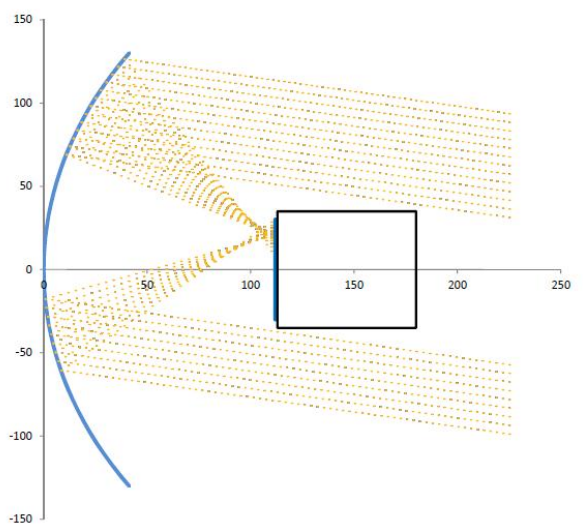

Figure 2: Modeling rays (yellow points) from a distant point source in the telescope. Blue curves illustrate the mirror and photocathode surface. Black rectangle imitates shadowing by the PMT case.
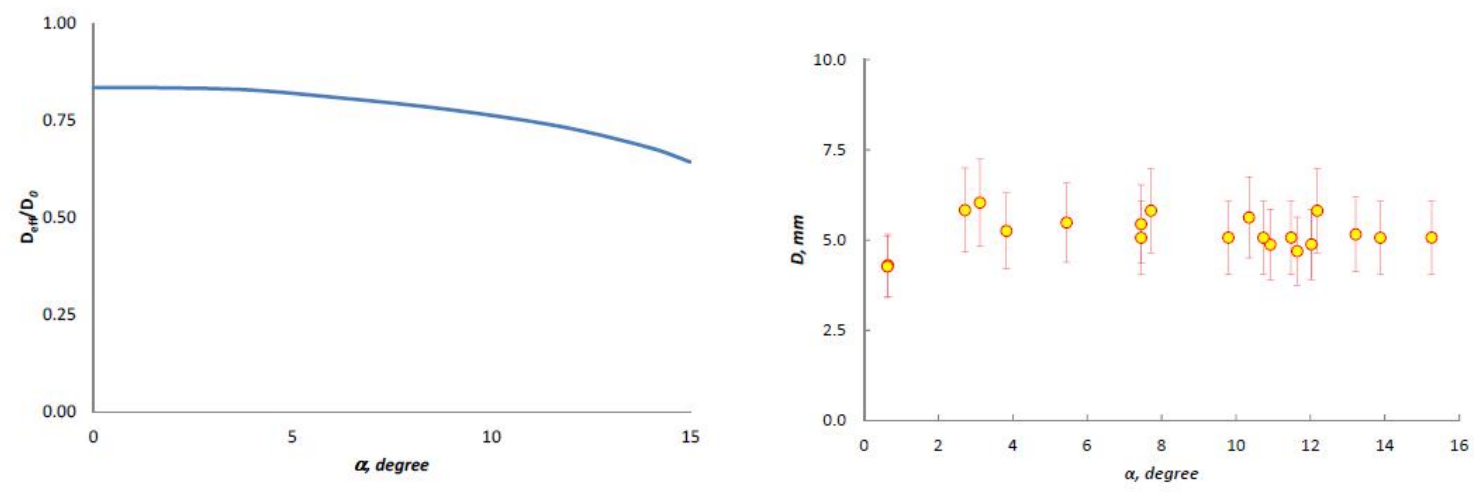

Figure 3: Right: Shadowing of the mirror by the PMT and support + cables. The ratio of unshielded effective mirror diameter to the actual diameter is given as a function of incidence angle, $\alpha$. Left: Fuzzy image diameter of the distant point source on the photocathode surface as a function of incidence angle, $\alpha$.

\section{Operation Algorithm}

The wide FoV cherenkov telescope consists of the spherical mirror and multi-anode PMT as an imaging camera in the focus. Full description of PMT [1]. Data acquisition system (DAQ) includes 32 operational amplifiers and amplitude-to-digital converters (ADCs) connected to the industrial PC. To model the focusing of the aluminized spherical mirror in the wavelength interval $(300,600)$ $\mathrm{nm}$ we have used a point source of light placed at infinity, with angle between the line to source and optical axis of the mirror. The image of the point source is calculated on the target plane near the focus of the mirror. The scheme of ray tracing is illustrated in (Fig. 2), where the spherical aberration of the point source image is seen on the PMT photocathode surface[2][3].

Since the photocathode Hamamatsu R2486 radius equal $3 \mathrm{~cm}$, mirror diameter was chosen equal $26 \mathrm{~cm}$ with $22.5 \mathrm{~cm}$ radius of curvature. We have found the optimal parameters of the telescope from the viewpoint of providing as wide field of view as possible, but with distortion not exceeding the pixel size $d=3.8 \mathrm{~mm}$. As a result, the width of the field of view was $-14^{0} \leq \alpha \leq 14^{0}$. PMT is used as an imaging camera and placed on the focal length in front of a spherical mirror. 


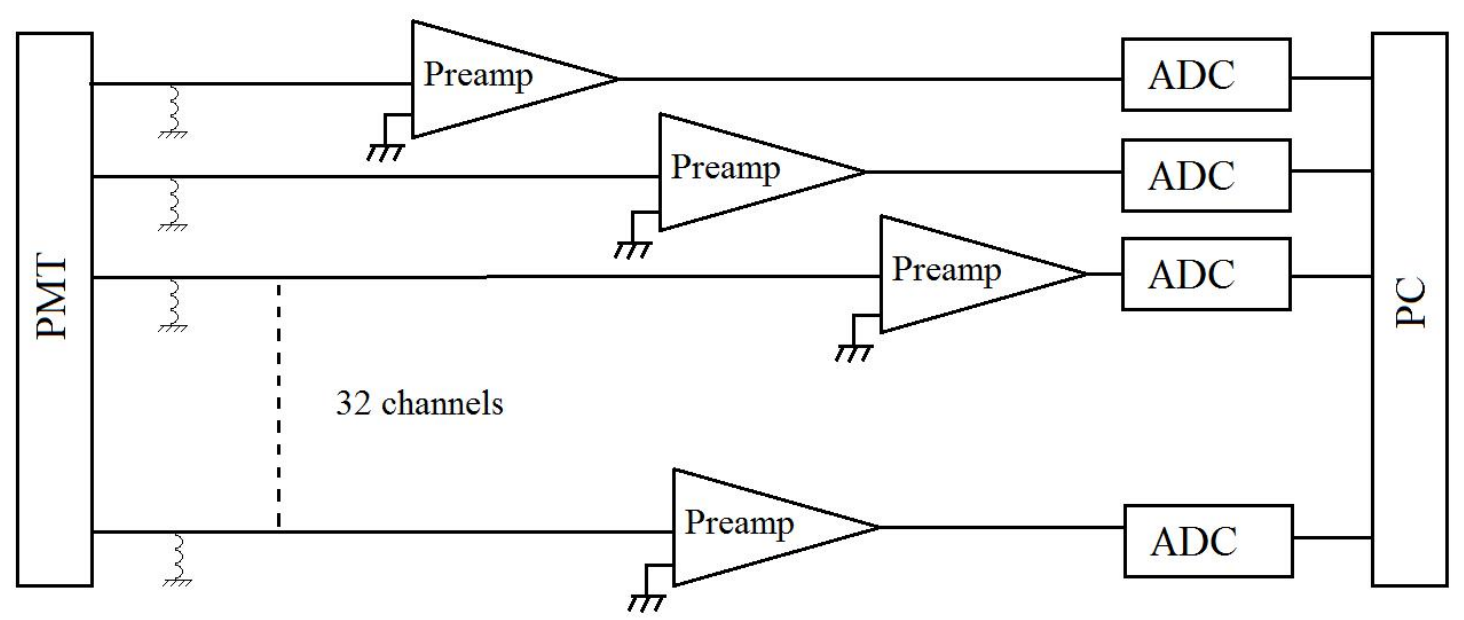

Figure 4: Data acquisition system flowchart

In this design telescope provides the effective aperture Deff $(00)=10,9 \mathrm{~cm}$ due to shadowing of the mirror by the PMT and support. Angular dependence of the telescope aperture is given in (Fig. 3) right. We calculated it through a ratio of the light intensity on the photocathode surface to the initial intensity falling into actual aperture of the telescope, taking into account the reflectance of aluminium, $92.4 \%$, in the PMT sensitivity interval 300-600 nm. The quality of the optical system is characterized by the spot size, where the spot is an image of the point source at infinity, on the focal plane. We have measured the spot size of the image on the photocathode formed by the laser pointer at $3 \mathrm{~m}$ from the telescope. Angular dependence of the spot size is shown in (Fig. 3) left. It is approximately consistent with results of our modeling. Corresponding angular resolution of the telescope is $\sim 1.40$ within FoV.

\section{Data Acquisition System}

The signals from multichannel photomultiplier continuously get to the preamplifier and then converting by ADC with a sampling frequency of $250 \mathrm{MHz}$ and stored in the buffer memory $(16 \mu \mathrm{s})$ 32-channel industrial PC OCZS-32-250USB (Industrial PC description can be found in [4]). On the arrival of a master signal generated by the Yakutsk EAS array, industrial computer keeps a buffer of accumulated pre-history in a file marked time and assignment of the serial number. Yakutsk EAS array description can be found in [5][6]. The flowchart of such system is shown in (Fig. 4).

Preamplifier transmission ratio is compatible with a long fiber line is estimated as 2.9. Measurement of dependence between transmission ratio and the power supply voltage of preamplifier $(5,10 \mathrm{~V})$ are not exceed noise level $\pm 6 m \mathrm{~V}$. Measurement of voltage induction between channels of preamplifiers are not exceed $\pm 6 \mathrm{mV}$. Coordinate-dependent sensitivity of the photomultiplier in range from $900 \mathrm{~V}$ to $1300 \mathrm{~V}$ is shown on (Fig. 5), which shows the radial non-uniformity of sensitivity[5][6]. Also we found that the amplitude of the dark current of the PMT at a voltage less than $1100 \mathrm{~V}$ lies within $\pm 6 m V$ range. 


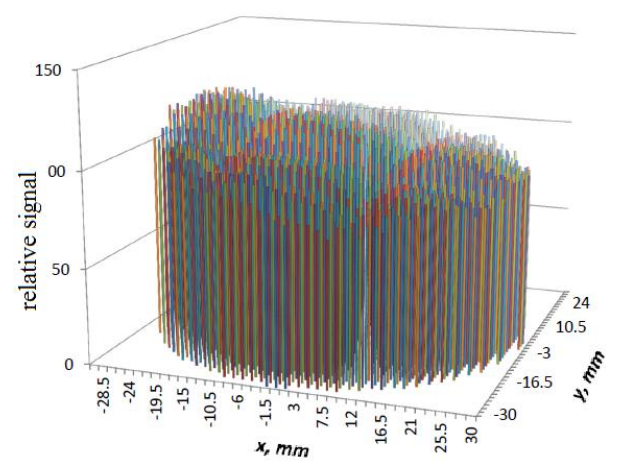

Figure 5: Normalized coordinate-dependent sensitivity of the photomultiplier
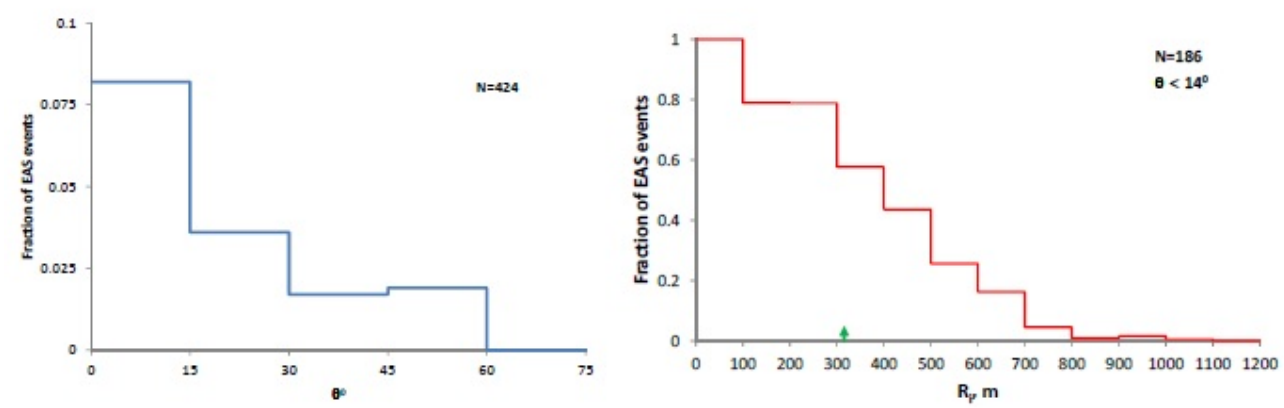

Figure 6: Zenith angle and axis distance distribution of the shower fraction detected with telescope

\section{First results of EAS measurements with Cherenkov telescope working in coincidence with surface detectors}

There has not been measurement of EAS Cherenkov light, with respect to the angular distribution, up to now, with one exception. The project is based on the idea of creating a small copy of the giant telescopes used in gamma-ray astronomy. The latest developments of photomultiplier tubes 


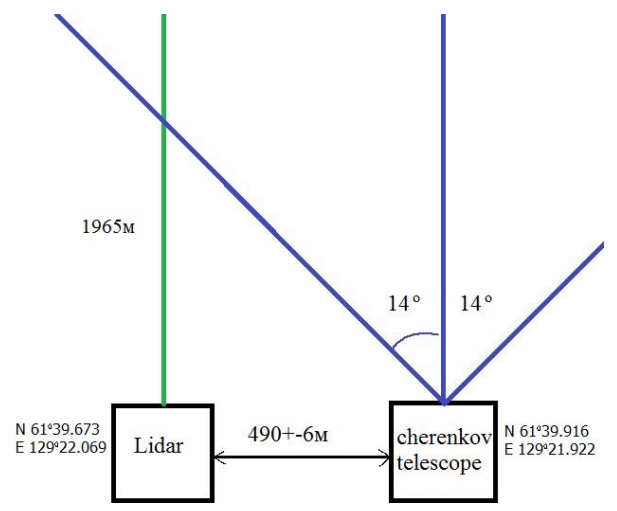

Figure 7: LIDAR signal entering into the aperture of the telescope flowchart

(PMT) allow to dramatically reducing the size of the Cherenkov telescope with the same angular resolution, which greatly reduces the final cost of the telescope. The energy work range $10^{16} \mathrm{eV}$ compensates reduction in the size of mirror.

During the field testing of the telescope in the period from 19.10.2012 to 11.04.2013 we had 604 hours of clear moonless nights, 11124 EAS events detected with scintillator subset of the array, from which 424 gave nonzero simultaneous signal in the telescope. Additional 277 telescope signals are triggered by the Cherenkov detectors; they will be analyzed later.

From numbers above (and the array area $8.2 \mathrm{~km} 2$ ) we got the effective radius of the telescope acceptance Reff $=315 \mathrm{~m}$. It can be used in planning of the detectors grid. The fraction of EAS events detected with nonzero telescope signal is only 3,8 shower axis distance is illustrated in Fig. 1; primary energies in the region E0 $>10^{16} \mathrm{eV}$. While the telescope FOV is $308 \mathrm{sq}$. degrees $(\theta \in$ $\left.\left(0^{0} ; 14^{0}\right)\right)$, EAS events are detected with zenith angles up to $\theta=60^{\circ}$. It can be explained by the broad angular distribution of emitting electrons in the shower and photon scattering in the atmosphere. Another contribution is angular uncertainty of the shower reconstruction procedure which is considerably increased at the lower threshold energy of the array. To avoid this uncertainty, we selected showers within $\theta<14^{0}$ drawing a distribution of the shower axes (Fig. 6). The effective radius of the telescope detecting area, Reff , is indicated by the arrow on the Ri axis as well. For the telescope was designed external safe box (Fig. 9).

Currently, the telescope is set at the Yakutsk EAS array, near the station obscura-3 (500 m from the center of the array), where the telescope has completed field testing. Also, we were able to detect the LIght Detection and Ranging (LIDAR) signal, located at a distance from the Cherenkov telescope. The distance between the LIDAR and the Cherenkov telescope measured by GPS is equal $490 \pm 6 \mathrm{~m}$.

The average LIDAR signal for 95 events shows that the signal amplitude dramatically increases when entering into the aperture of the telescope height $2 \mathrm{~km}$ than reaching a maximum value and attenuation according to the square of the distance. The obtained data indirectly confirms the proper functioning of the Cherenkov telescope as a measuring device. 


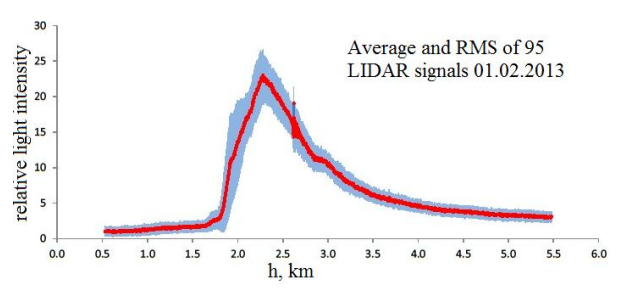

Figure 8: Average and RMS of 95 LIDAR signals 01.02.2013

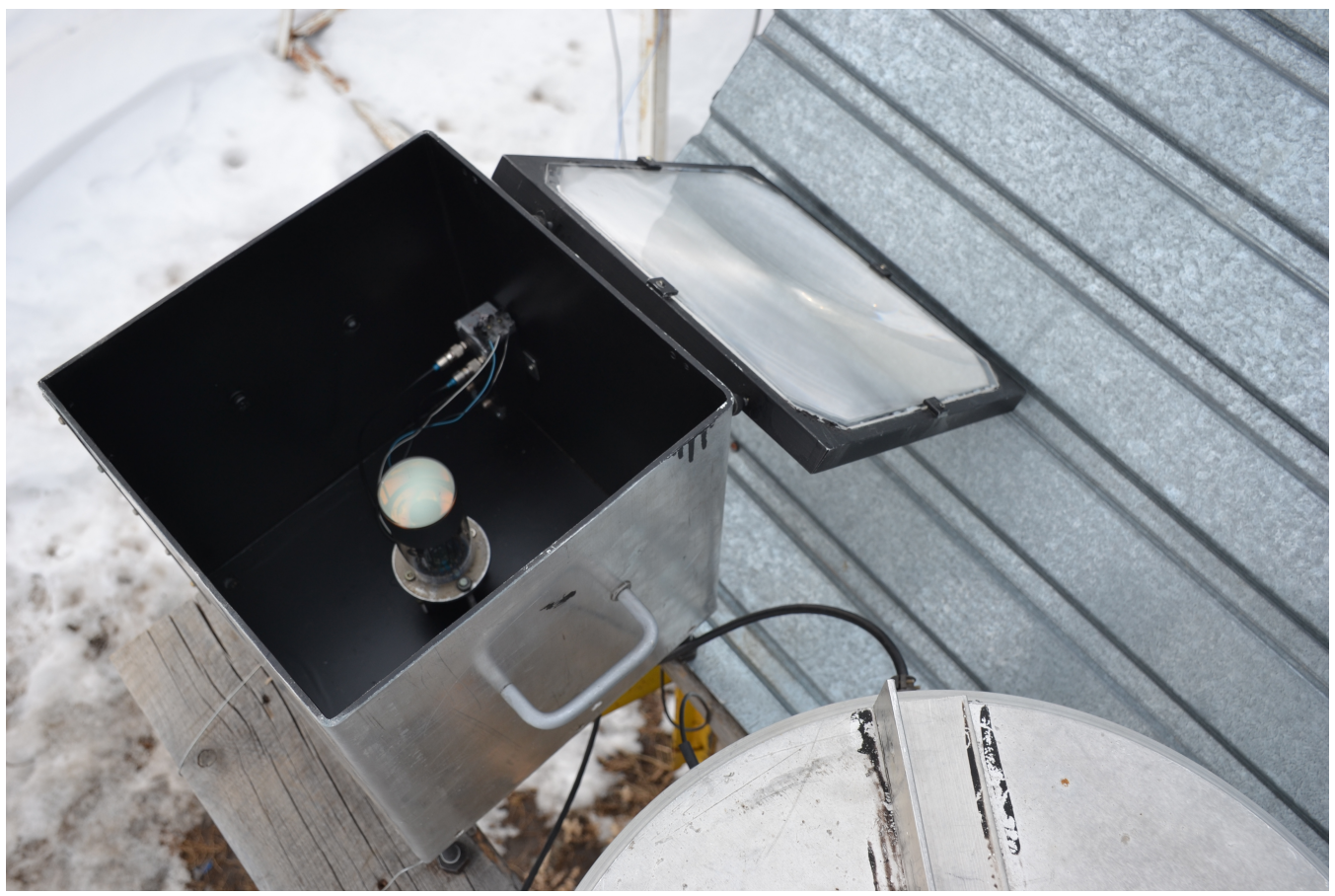

Figure 9: Integral telescope prototype

\section{Further prototype}

During field-testing of the telescope it proved that define Xmax is a much more precise on time characteristics than the angular so we have come to a new concept of Cherenkov telescope (Fig. 9). We produced two prototypes based on fast inegral Enterprise PMT, $28 \mathrm{~cm}$ Fresnel lens and $1 \mathrm{GHz}$ Domino Ring Sampler Evaluation Board as ADC that were field tested in 10 moonless nights. At the moment we are taking angular-dependent sensitivity and dark current limit. Measurement of voltage induction between channels of preamplifiers is not exceeding $\pm 6 \mathrm{mV}$.

We have developed and built two experimental prototypes: a fast integral Cherenkov telescope and a wide FoV Cherenkov telescope working in collaboration with scintillation detectors, inte- 
gral and differential Cherenkov detectors of the Yakutsk EAS. Cherenkov telescope successfully completed field testing in the period from 19.10.2012 to 25.01.2015 we had 1369 hours of clear moonless nights, 80000 EAS events detected with scintillator subset of the array, from which 1000 gave nonzero simultaneous signal in the telescope. Analyzing this experimental data can sufficiently exceed possibilities of Yakutsk EAS array to determine the parameters of galactic cosmic rays.

Acknowledgment:The work is supported in part by RFBR (grants 11-02-00158, 11-02-12193, 12-02-10005, 1202-31550) and the Russian Ministry of Education and Science (contracts 02.740.11.0248, 16.518.11.7075).

\section{References}

[1] http://www.hamamatsu.com/

[2] A.A. Ivanov et al., Astrophys.Space Sci.Trans. 6 (2010) 53 doi:10.5194/astra-6-53-2010.

[3] A.A. Ivanov et al., J. Phys.: Conf. Ser., 409 (2013) 012084 doi:10.1088/1742-6596/409/1/012084.

[4] http://www.rudshel.ru/

[5] A.A. Ivanov, S.P.Knurenko and I.Ye.Sleptsov, J. Exp. Theor. Phys. 104 (2007) 870 doi:10.1134/S1063776107060052.

[6] A.A. Ivanov, S.P. Knurenko and I.Ye. Sleptsov, New J. Phys. 11 (2009) 065008 doi:10.1088/1367-2630/11/6/065008. 